\title{
M-CSF neutralization and Egr-1 deficiency prevent ovariectomy-induced bone loss
}

\author{
Simone Cenci, ${ }^{1,2}$ M. Neale Weitzmann, ${ }^{1}$ M.A. Gentile, ${ }^{3}$ M.C. Aisa, ${ }^{1}$ and Roberto Pacifici ${ }^{1}$ \\ ${ }^{1}$ Division of Bone and Mineral Diseases, Washington University School of Medicine and Barnes-Jewish Hospital, \\ St. Louis, Missouri, USA \\ ${ }^{2}$ Department of Gerontology and Geriatrics, University of Perugia, Perugia, Italy \\ ${ }^{3}$ Department of Bone Biology/Osteoporosis, Merck Research Laboratories, West Point, Pennsylvania, USA
}

Address correspondence to: Roberto Pacifici, Division of Bone and Mineral Diseases, Barnes-Jewish Hospital, North Campus, 216 South Kingshighway, St. Louis, Missouri 63110, USA.

Phone: (314) 454-8407; Fax: (314) 454-5047; E-mail: Pacifici@im.wustl.edu.

Received for publication October 14, 1999, and accepted in revised form March 27, 2000.

Increased stromal cell production of M-CSF, an event caused by enhanced phosphorylation of the nuclear protein Egr-1, is central to the mechanism by which estrogen (E2) deficiency upregulates osteoclast (OC) formation. However, the contribution of enhanced M-CSF production to the bone loss induced by $\mathrm{E} 2$ deficiency remains to be determined. We found that treatment with an $\mathrm{Ab}$ that neutralizes M-CSF in vivo completely prevents the rise in OC number, the increase in bone resorption, and the resulting bone loss induced by ovariectomy (ovx). We also found that adult, intact Egr-1-deficient mice, a strain characterized by maximally stimulated stromal cell production of M-CSF, exhibit increased bone resorption and decreased bone mass. In these mice, treatment with anti-M-CSF Ab restored normal levels of bone resorption, thus confirming that increased M-CSF production accounts for the remodeling abnormalities of Egr-1-deficient mice. Consistent with the failure of ovx to further increase M-CSF production in Egr-1-deficient mice, ovx neither increased bone resorption further, nor caused bone loss in these animals. In summary, the data demonstrate that $\mathrm{E} 2$ deficiency induces M-CSF production via an Egr-1-dependent mechanism that is central to the pathogenesis of ovx-induced bone loss. Thus, Egr-1 and M-CSF are critical mediators of the bone sparing effects of $\mathrm{E} 2$ in vivo.

J. Clin. Invest. 105:1279-1287 (2000).

\section{Introduction}

It is now recognized that one of the main mechanisms by which estrogen (E2) deficiency causes bone loss is by stimulating osteoclast (OC) formation (1), a process facilitated by bone marrow stromal cells (SC). SC provide a physical support for nascent OCs and produce soluble and membrane-associated factors that regulate the proliferation and differentiation of OC precursors (2). Among these factors are osteoprotegerin ligand (OPGL, also known as RANKL, TRANCE, or ODF) (3-5) and M-CSF (6-8). Whereas neither of these two factors is capable of inducing $\mathrm{OC}$ formation in the absence of the other, together they induce the differentiation of hematopoietic precursors of the monocytic lineage into mature OCs capable of resorbing bone (3, $5)$. The formation of mature OCs is completely dependent on the presence of both OPGL and M-CSF, as demonstrated by the absence of OC development in mice lacking the expression of either factor $(4,9,10)$.

Evidence has also accumulated that suggests that MCSF plays a key role in enhancing the production of OCs in conditions of E2 deficiency. We have shown that SC from ovariectomized (ovx) mice produce larger amounts of soluble M-CSF than SC from E2-replete mice, a phenomenon that increases the osteoclastogenic activity of SC (11). Ovx leads to the generation of SC characterized by enhanced casein kinase II-depend- ent (CKII-dependent) phosphorylation of the nuclear protein Egr-1. Phosphorylated Egr-1 binds less avidly than dephosphorylated Egr-1 to the transcriptional activator $\mathrm{Sp}-1$, resulting in higher levels of free $\mathrm{Sp}-1$ that stimulate transactivation of the M-CSF gene (12). Thus, Egr-1 is a key mediator of the mechanism by which E2 regulates M-CSF production in SC. Attesting to the relevance of Egr-1 as a regulator of M-CSF production in vivo, we have found that $\mathrm{E} 2$ replacement fails to block M-CSF production and OC formation in ovx mice lacking Egr-1 (12).

Other investigators have reported that E2 downregulates the bone marrow cell production of membranebound M-CSF, a phenomenon that contributes to repress OC formation and bone resorption. $(13,14)$. These data and reports indicating that M-CSF increases OC survival and chemotactic behavior of isolated OCs (15), suggest that increased production of M-CSF accounts, at least in part, for the increased bone resorption and the resulting bone loss that occur in E2-deficient animals. However, the contribution of M-CSF to the pathogenesis of ovx-induced bone loss remains to be elucidated, because E2 regulates the production of several cytokines recognized as potent inducers of osteoclastogenesis and bone resorption. Among these factors are IL-1, IL-6, and TNF (1, 16-18). Moreover, E2 could also decrease 
OPGL-induced osteoclastogenesis, because it stimulates the production of the decoy OPGL receptor osteoprotegerin (19).

In this study, we have used two experimental models to investigate the role of M-CSF as a cause of ovxinduced bone loss. First, we have determined whether in vivo treatment with an antibody that neutralizes $M$ CSF prevents bone loss in ovx wild-type (WT) mice. Second, we have assessed the effects of ovx and E2 replacement on Egr-1-deficient mice.

We report that in vivo treatment with anti-M-CSF antibody completely prevents ovx-induced bone loss. We also demonstrate that neither ovx nor E2 replacement induce changes in the bone density of Egr-1-deficient mice, thus establishing that a key mechanism by which E2 deficiency induces bone loss involves Egr1-regulated production of M-CSF.

\section{Methods}

All animal procedures were approved by the Animal Care and Use Committee of Barnes-Jewish Hospital. Unless otherwise specified, reagents and media were purchased from the Sigma Chemical Co., St. Louis, Missouri, USA.

Study protocol. To determine if neutralization of MCSF prevents ovx-induced bone loss, 4-month-old Swiss Webster mice (Taconic Farms, Germantown, New Jersey, USA) were ovx or sham-operated by the dorsal approach under general anesthesia $(11,20)$. Ovx mice were treated with either the $\mathrm{mAb} 5 \mathrm{~A} 1$, which specifically neutralizes murine M-CSF (21) (developed and provided by H.S. Lin, Washington University, St. Louis, Missouri, USA), an irrelevant Ab of the same isotype, or $17 \beta$ estradiol for the first 4 weeks after surgery. The Ab 5A1 and the irrelevant antibody were injected intraperitoneally at a dose of $0.5 \mathrm{mg} /$ week. At this dose $5 \mathrm{~A} 1 \mathrm{Ab}$ is known to neutralize the biological functions of M-CSF in vivo (22). The in vivo neutralizing activity of sera from $5 \mathrm{~A} 1 \mathrm{Ab}$-treated mice was confirmed by demonstrating the ability of sera, collected at the end of the treatment period, to block the MCSF-induced proliferation and survival of murine monocytes $\left(\mathrm{CD} 11 \mathrm{~b}^{+}\right.$cells) in vitro. Implanted in a nuchal subcutaneous pocket, $17 \beta$ estradiol (0.16 $\mu \mathrm{g} /$ day, the lowest dose that maintains a normal uterine weight) was administered using slow-release subcutaneous pellets (Innovative Research of America, Toledo, Ohio, USA).

In a second series of experiments, we investigated the effects of ovx and E2 replacement in Egr-1 knock-out (Egr-1-/-) mice (23) and age-matched WT control littermates of the same genetic background, a kind gift of Jeffrey Milbrandt (Washington University). Genetic homogeneity was obtained by back breeding the original heterozygous mice $($ Egr-1+/-) into the background strain $(\mathrm{C} 57 \mathrm{Bl} / 6)$ for 10 generations.

Homozygous mice were then generated by crossbreeding male and female mice, each heterozygous for Egr-1. Body weight, size, and E2 serum levels of the Egr$1^{-/-}$mice used in this study were indistinguishable from those of WT littermates (24). However, E2 treatment fails to decrease SC production of M-CSF and in vitro OC formation in ovx Egr-1/- mice (12).

A first group of intact Egr-1/- mice and WT control littermates were sacrificed at the age of 4 months to collect sera for osteocalcin analysis. A second group of mice was used to carry out an experiment in which intact Egr-1-/mice and control littermates of 4 months of age were treated with either $5 \mathrm{~A} 1 \mathrm{Ab}$ or irrelevant $\mathrm{Ab}$ for 4 weeks, as described above. A third group of Egr-1 $1^{-/-}$mice and WT control littermates of 4 months of age were either sham-operated or ovx as described above. The shamoperated mice were left untreated, whereas ovx mice were treated with either control vehicle or $17 \beta$ estradiol ( 0.16 $\mu \mathrm{g} /$ day) for 4 weeks, as described above.

Atrophy of the uterus was demonstrated at sacrifice in all ovx mice, except those treated with E2, thus confirming that ovx was successful in blunting circulating E2 levels. Both at baseline and at the end of each study, there were no differences in body weight between ovx and sham-operated mice and no differences between treatment groups.

Bone density measurements. Bone density was measured by dual-energy x-ray absorptiometry (DEXA) performed on a QDR-2000 Plus Densitometer (Hologic, Bedford, Massachusetts, USA). The machine was calibrated daily with a hydroxyapatite phantom of the murine spine. Anesthetized mice were supine and positioned with tape on limbs on a precision-milled acrylic block. Tape was also used on the abdomen and neck to ensure maximum adherence of spine and pelvis to the surface. Measurements were made with a customized mouse whole body software package (Hologic). The global window was defined as the whole body image minus the skull, mandible, and teeth $(175 \times 135$ pixels $)$. Two regions of interest were generated, corresponding to the right (R1) and the left rear limb (R2). For each measurement, a limb box included the femur, tibia, and ankle. The scans were done with a $1.270-\mathrm{mm}$-diameter collimator, 0.762 $\mathrm{mm}$ line spacing, $0.380-\mathrm{mm}$ point resolution, and an acquisition time of 7 minutes. The bone mineral density (BMD) of the right and the left limb were averaged and expressed in milligrams per square centimeter. Each mouse was scanned three times at each time point, with repositioning between scans. The data presented are the average of the three measures. Data from the triplicate measures of each mouse obtained at the initial time point were used to determine the short-term reproducibility of the method. The mean difference in BMD between the three measurements was not significantly different from zero, and the mean coefficient of variation was $2.0 \%$. Long-term reproducibility was assessed by repeated measurements of the murine phantom. Serial scans were analyzed using the same regions of interest as in the first scan for all subsequent scans and using dedicated software for longitudinal comparisons.

Bone bistomorphometry. All animals were injected intraperitoneally with calcein $(30 \mathrm{mg} / \mathrm{kg}$ body weight) 19 and 26 days after surgery. Four weeks after surgery 
mice were sacrificed and tibiae were removed and fixed in $70 \%$ ethanol. The proximal $8 \mathrm{~mm}$ was trimmed with a Dremel tool, dehydrated, and embedded undecalcified in methyl methacrylate, as described previously $(25,26)$. Longitudinal sections $(5 \mu \mathrm{m}$ thick) taken in the frontal plane through the cancellous bone of the proximal tibia were prepared with a Reichert-Jung Polycut microtome, mounted on glass slides, and stained with Goldner's trichrome. Coverslips were affixed with Eukitt's mounting medium. Histomorphometric analysis was performed using Bioquant True Colors for Windows (R \& M Biometrics Inc., Nashville, Tennessee, USA) by an investigator blind to type of surgery and treatment. Measurements were obtained in an area of cancellous bone that measured approximately 2.5 $\mathrm{mm}^{2}$, contained only secondary spongiosa, and was located $0.5-2.5 \mathrm{~mm}$ distal to the epiphyseal growth cartilage. Total perimeter (Tt.Pm) of cancellous bone surface was measured at $\times 40$. Osteoclast perimeter (Oc.Pm), a standardized index of bone resorption, osteoblast surface (OS/BS), a static index of bone formation, and bone formation rate (BFR/BS), a dynamic (tetracycline-based) index of bone formation, were measured at $\times 200$. OC surface (Oc.S/BS) was calculated as a percentage (Oc.Pm/Tt.Pm). BFR/BS was also measured in cancellous bone of the femoral metaphysis excluding cortical bone on $5-\mu \mathrm{m}$ thick longitudinal sections. All the indices were defined according to the American Society of Bone and Mineral Research histomorphometry nomenclature (27).

Deoxypyridinoline crosslinks assay. The urinary excretion of deoxypyridinoline (DPD) crosslinks, a marker of bone resorption (28), was measured in urine samples using the ELISA kit developed and kindly provided by Metra Biosystems (Mountain View, California, USA) (29). Results were expressed as nanomole per millimole of urinary creatinine ( $\mathrm{Cr}$ ), as measured by a standard colorimetric technique. The intra- and the inter-assay variation of this method were less than $9 \%$ and $15 \%$, respectively (30).

Osteocalcin assay. Serum osteocalcin levels were measured using the competitive RIA assay kit by Biomedical Technologies Inc. (Stoughton, Massachusetts, USA). The intra- and the inter-assay variation of this method were less than $6 \%$ and $12 \%$, respectively.

Assessment of serum neutralization activity. The ability of $5 \mathrm{~A} 1 \mathrm{Ab}$ to neutralize M-CSF in vivo was assessed by demonstrating that sera harvested at sacrifice from mice treated with $5 \mathrm{~A} 1 \mathrm{Ab}$ blocks the M-CSF-dependent proliferation of $\mathrm{CD} 11 \mathrm{~b}^{+}$cells.

Sera $(100 \mu \mathrm{L})$ from mice treated with either $5 \mathrm{~A} 1 \mathrm{Ab}$ or irrelevant $\mathrm{Ab}$ and fresh $5 \mathrm{~A} 1 \mathrm{Ab}(200 \mathrm{ng}$ in $100 \mu \mathrm{L})$ were serially diluted (1:2) and added to bone marrow $\mathrm{CD}_{11} \mathrm{~b}^{+}$cells (final volume $200 \mu \mathrm{L}$ per well) purified by positive immunomagnetic selection using a commercial kit (Miltenyi Biotec Inc., Auburn, California, USA). Recombinant mouse M-CSF $(25 \mathrm{ng} / \mathrm{mL})$ was added to each well. At the end of a 3-day culture period cell numbers were measured using the MTT assay (31).
Statistical analysis. The effects of surgery and treatment on BMD was assessed by ANOVA for repeated measurements. Subsequent multiple comparison tests were performed by the Fisher protected least significant difference test. Group mean values were compared using 2-tailed Student's $t$ test, or 1-way analysis of variance and Fisher protected least significant difference test, as appropriate.

\section{Results}

Sera from mice treated with anti-M-CSF antibody neutralizes $M$-CSF in vivo. When tested in a proliferation assay, the serum of mice treated with $5 \mathrm{~A} 1 \mathrm{Ab}$ for 4 weeks inhibited M-CSF-dependent survival and proliferation of $\mathrm{CD}_{11} \mathrm{~b}^{+}$cells in a manner similar to fresh recombinant $5 \mathrm{~A} 1 \mathrm{Ab}$ (Figure 1). In these experiments, parallel and dose-responsive inhibition curves of M-CSF-induced monocyte proliferation were obtained with fresh 5A1 $\mathrm{Ab}$ and sera from $5 \mathrm{~A} 1 \mathrm{Ab}$-treated mice. The sample dilution containing either $6.25 \mu \mathrm{L}$ serum or $12.5 \mathrm{ng}$ fresh $5 \mathrm{~A} 1 \mathrm{Ab}$ per well inhibited $50 \%$ of the M$\mathrm{CSF}$-induced proliferation. Serum from mice treated with irrelevant $\mathrm{Ab}$ had no effect on M-CSF-induced monocyte proliferation. These findings demonstrate that $5 \mathrm{~A} 1 \mathrm{Ab}$ neutralizes $\mathrm{M}-\mathrm{CSF}$ in vivo.

Treatment with anti-M-CSF antibody prevents ovx-induced bone loss. To determine if M-CSF neutralization prevents ovx-induced bone loss, mature Swiss Webster

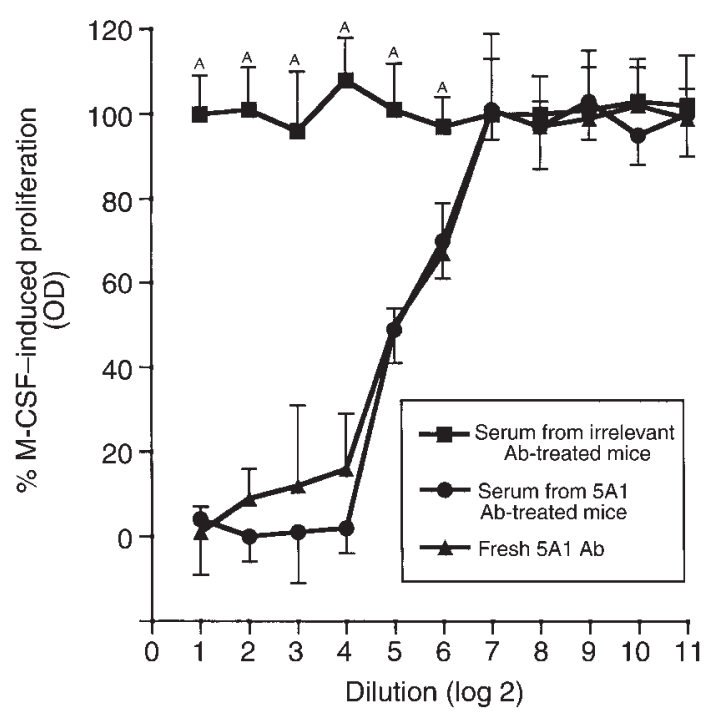

\section{Figure 1}

Effect of serum from 5A1 Ab-treated mice on M-CSF-induced monocyte proliferation. Results (mean \pm SEM) are expressed as percent of M-CSF-induced proliferation. Sera $(100 \mu \mathrm{L})$ obtained at the end of the treatment period from ovx mice treated with either $5 \mathrm{~A} 1 \mathrm{Ab}$ or irrelevant $A b$ and fresh $5 A 1 \mathrm{Ab}(200 \mathrm{ng}$ in $100 \mu \mathrm{L})$ were serially diluted $(1: 2)$ and added to $C D 11 b^{+}$cells seeded in triplicate in 96-well plates (final volume $200 \mu \mathrm{L}$ per well). Recombinant murine M-CSF $(25 \mathrm{ng} / \mathrm{mL})$ was then added to each well. Cell proliferation was measured after a 3-day incubation, as described in Methods. ${ }^{A} P<0.05$, compared with the other groups. 


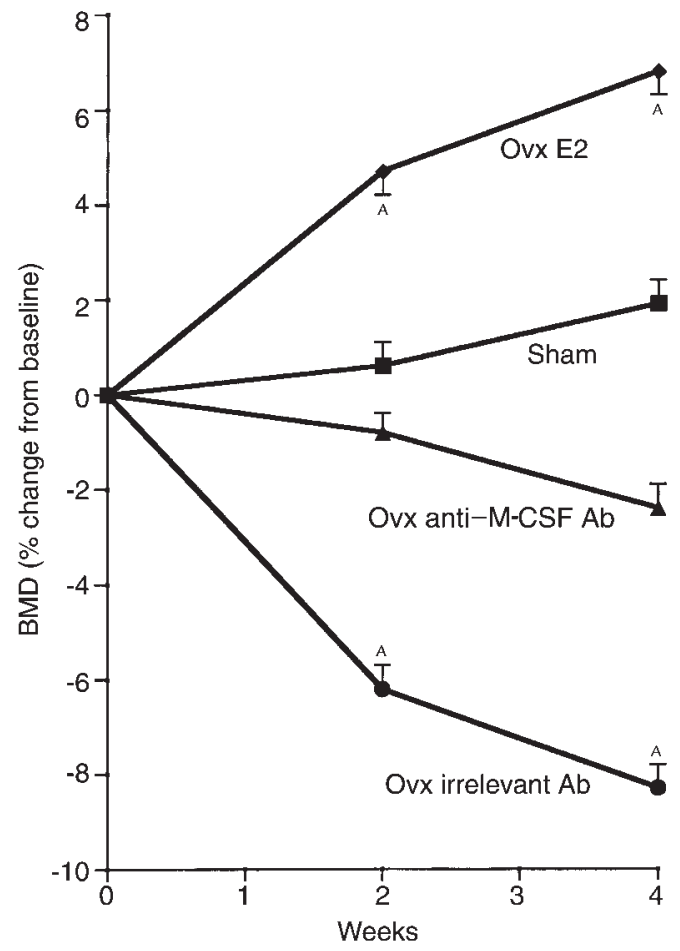

Figure 2

Treatment with $5 \mathrm{~A} 1 \mathrm{Ab}$ prevents ovx-induced bone loss. Results (mean \pm SEM) are expressed as percent of change from baseline. Mice 4 months of age were ovx or sham-operated. Ovx mice were treated with either the anti-M-CSF Ab 5A1, an isotype-matched irrelevant $A b$, or E2 for the first 4 weeks after surgery. Sham-operated mice were left untreated. In vivo measurements of rear-limb BMD were carried out by DEXA as described in Methods before surgery and 2 and 4 weeks after surgery ( $n=10$ mice per group). Baseline BMD values were as follows: Sham, $62.5 \pm 1.0 \mathrm{mg} / \mathrm{cm}^{2}$; ovx-irrelevant Ab, $61.9 \pm 0.6$ $\mathrm{mg} / \mathrm{cm}^{2}$; ovx E2, $62.8 \pm 0.9 \mathrm{mg} / \mathrm{cm}^{2}$; ovx $5 \mathrm{~A} 1 \mathrm{Ab}, 63.6 \pm 1.0 \mathrm{mg} / \mathrm{cm}^{2}$. There was no significant difference for baseline BMD among groups. ${ }^{A} P<0.05$ compared with baseline and with any other group.

mice were either sham-operated or ovx. Sham-operated mice were left untreated whereas ovx mice were treated with either irrelevant $\mathrm{Ab}, 5 \mathrm{~A} 1 \mathrm{Ab}$, or E2. All treatments were started the day of ovx and continued for 4 weeks. BMD measurements of the rear limbs were obtained before, as well as 2 and 4 weeks after, surgery.

At baseline all groups of mice had similar BMD values (Figure 2). During the 4 weeks of follow-up, BMD did not change significantly in sham-operated mice. Consistent with the known potent anabolic effect of E2 in Swiss Webster mice (32), BMD increased $6.8 \%$ over baseline $(P<0.05)$ in E2-treated ovx mice. In contrast, BMD decreased rapidly in ovx mice treated with irrelevant antibody. Four weeks after surgery BMD was, in this group, $8.3 \%$ lower than at baseline $(P<0.05)$. Treatment with $5 \mathrm{~A} 1 \mathrm{Ab}$ almost completely prevented the bone loss induced by ovx. In fact, at 4 weeks after surgery, the BMD of ovx mice treated with $5 \mathrm{~A} 1 \mathrm{Ab}$ was only $2.4 \%$ lower than at baseline $(P=\mathrm{NS})$ and higher $(P<0.05)$ than that of ovx mice treated with irrelevant $\mathrm{Ab}$. These data demonstrate that M-CSF neutralization prevents ovx-induced bone loss.
Treatment with anti-M-CSF antibody prevents the increase in bone turnover induced by ovx. To determine if M-CSF neutralization prevents the increase in bone resorption induced by ovx, the urinary excretion of DPD crosslinks, a marker of bone resorption, was measured before and 2 and 4 weeks after surgery. At baseline all groups of mice had similar DPD excretion levels (Figure 3). Whereas sham operation was not followed by a significant change in DPD excretion ( $+3.9 \%$ compared with baseline, $P=$ NS), ovx caused a $31 \%$ increase in DPD excretion $(P<0.05)$, as compared with baseline. This increase was blunted by treatment with either anti-M-CSF antibody $(+12 \%$ compared with baseline, $P=\mathrm{NS})$ or E2 ( $+4 \%$ compared with baseline, $P=\mathrm{NS})$. Thus, at 4 weeks from surgery DPD levels were similar in sham-operated mice and ovx mice treated with either $\mathrm{E} 2$ or $5 \mathrm{~A} 1 \mathrm{Ab}$. These data demonstrate that M-CSF neutralization prevents the increase in bone resorption induced by $\mathrm{E} 2$ withdrawal.

To determine if $5 \mathrm{~A} 1 \mathrm{Ab}$ prevents the increase in histomorphometric indices of bone turnover that occurs after ovx, Oc.S/BS, an index of bone resorption, $\mathrm{OS} / \mathrm{BS}$, a static index of bone formation, and BFR/BS, a dynamic index of bone formation, were measured in cancellous bone of the tibia in five mice per group,

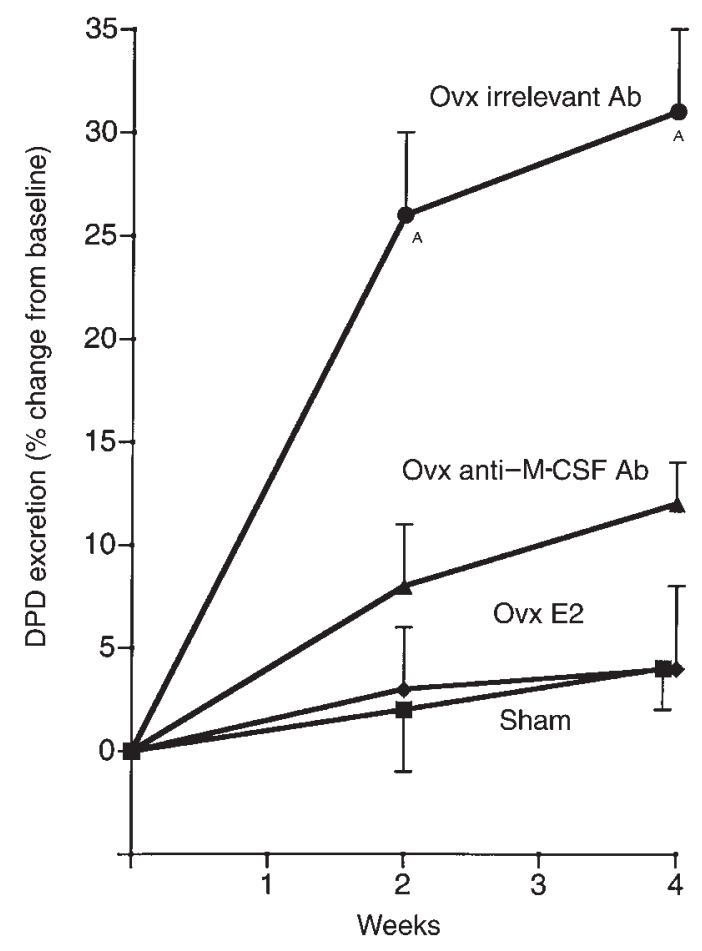

Figure 3

Treatment with $5 \mathrm{~A} 1 \mathrm{Ab}$ prevents the increase in DPD excretion induced by ovx. Results (mean \pm SEM) are expressed as percent of change from baseline. Mice were treated as described in Figure $1(n=10$ mice per group). Baseline DPD values were as follows: sham, $18.6 \pm 0.4$ $\mathrm{nmol} / \mathrm{mmol} \mathrm{Cr}$; ovx irrelevant $\mathrm{Ab}, 18.8 \pm 0.4 \mathrm{nmol} / \mathrm{mmol} \mathrm{Cr}$; ovx E2, $17.2 \pm 0.2 \mathrm{nmol} / \mathrm{mmol} \mathrm{Cr}$; ovx $5 \mathrm{~A} 1 \mathrm{Ab}, 19.9 \pm 0.4 \mathrm{nmol} / \mathrm{mmol} \mathrm{Cr}$. There was no significant difference for baseline DPD excretion among groups. ${ }^{A} P<0.05$ compared with baseline and with any other group. 
Table 1

Histomorphometric indices of bone turnover and serum osteocalcin levels in mice treated with estrogen and $5 \mathrm{~A} 1 \mathrm{Ab}$ at 4 weeks after surgery

\begin{tabular}{lcccc}
\hline & Sham & Ovx irrelevant Ab & Ovx E2 & Ovx 5A1 Ab \\
Oc.S/BS (\%) & $0.73 \pm 0.11$ & $2.29 \pm 0.16^{\mathrm{A}}$ & $0.68 \pm 0.39$ & $0.64 \pm 0.13$ \\
OS/BS $(\%)$ & $1.10 \pm 0.36$ & $2.50 \pm 1.00^{\mathrm{A}}$ & $0.81 \pm 0.36$ & $1.05 \pm 0.52$ \\
BFR/BS $\left(\mu \mathrm{m}^{3} / \mu \mathrm{m}^{2} / \mathrm{yr}\right)$ & $51.9 \pm 2.3$ & $95.6 \pm 4.7^{\mathrm{A}}$ & $58.3 \pm 2.7$ & $61.7 \pm 3.1$ \\
Osteocalcin $(\mathrm{ng} / \mathrm{mL})$ & $46.8 \pm 1.4$ & $60.4 \pm 1.5^{\mathrm{B}}$ & $45.8 \pm 1.4$ & $60.9 \pm 1.9^{\mathrm{B}}$
\end{tabular}

${ }^{A} P<0.05$ compared with each of the other groups. ${ }^{B} P<0.05$ compared with sham-operated mice.

randomly selected from the entire set of animals. However, in all groups the amount of tibial trabecular surface that exhibited double-tetracycline labeling was insufficient for obtaining a precise estimation of the rate of bone formation. Therefore, BFR/BS was measured in cancellous bone of the femoral metaphysis. We found that in the femora, the mineralized surface corresponded to the osteoid surface and that the amount of double labeling was sufficient for measuring BFR/BS.

Table 1 shows that bone resorption and bone formation were threefold and twofold higher, respectively, in ovx mice treated with irrelevant $\mathrm{Ab}$ than in both shamoperated mice and ovx mice treated with either E2 or $5 \mathrm{~A} 1 \mathrm{Ab}$. E2-replete mice and ovx mice treated with 5A1 $\mathrm{Ab}$ had similar indices of both bone resorption and bone formation. These findings demonstrate that $\mathrm{M}$ CSF neutralization prevents both the increase in OC number occurring after ovx and the changes in bone formation that are "coupled" to bone resorption.

To investigate the effects of M-CSF neutralization on osteoblast activity, the serum level of osteocalcin, a marker of bone formation, was measured in samples harvest$\mathrm{ed}$ at the end of the study. At 4 weeks from surgery, osteocalcin levels were approximately $33 \%$ higher $(P<0.05)$ in ovx mice treated with irrelevant $\mathrm{Ab}$ than in both shamoperated and E2-treated ovx mice (Table 1). The ovxinduced increase in osteocalcin was not prevented by MCSF neutralization, because its levels were similar in ovx mice treated with irrelevant $\mathrm{Ab}$ and $5 \mathrm{~A} 1 \mathrm{Ab}$. Since the increase in osteocalcin induced by ovx is due to upregulated osteoblastogenesis (33), these findings suggest that estrogen withdrawal upregulates osteoblast proliferation through an M-CSF-independent mechanism.

Egr-1-deficient mice have decreased bone density and increased bone resorption as result of increased M-CSF production. To investigate if Egr-1 deficiency alters skeletal development, BMD was measured every 2 weeks between the ages of 4 and 20 weeks in Egr-1/- mice and age-matched WT littermates of identical genetic background. This study revealed that BMD reaches a plateau at the age of 4 months in both Egr-1/- mice and control littermates (data not shown). However, at 4 months of age BMD was (Figure 4a) approximately $10 \%$ lower in Egr-1 ${ }^{-/}$than in WT mice $(P=0.002)$. DPD excretion was also $33 \%$ higher $(P<0.05)$ in Egr-1 $1^{-/}$than in WT mice (Figure $4 \mathrm{~b}$ ). These findings are consistent with the fact that Egr-1/- mice produce higher levels of M-CSF than WT controls (12). Interestingly, serum osteocalcin levels were $27 \%$ higher $(P<0.05)$ in Egr1-deficient mice than in control littermates (Figure 4c), a finding likely to reflect a compensatory increase in cancellous bone formation induced by the chronic stimulation of bone resorption.

To demonstrate that the increase in bone turnover of Egr-1-deficient mice is a result of increased M-CSF production, additional intact Egr-1/- mice and control littermates were treated for 4 weeks with either $5 \mathrm{~A} 1 \mathrm{Ab}$ or irrelevant antibody, as described above. Confirming the results of the previous study, baseline DPD excretion was higher
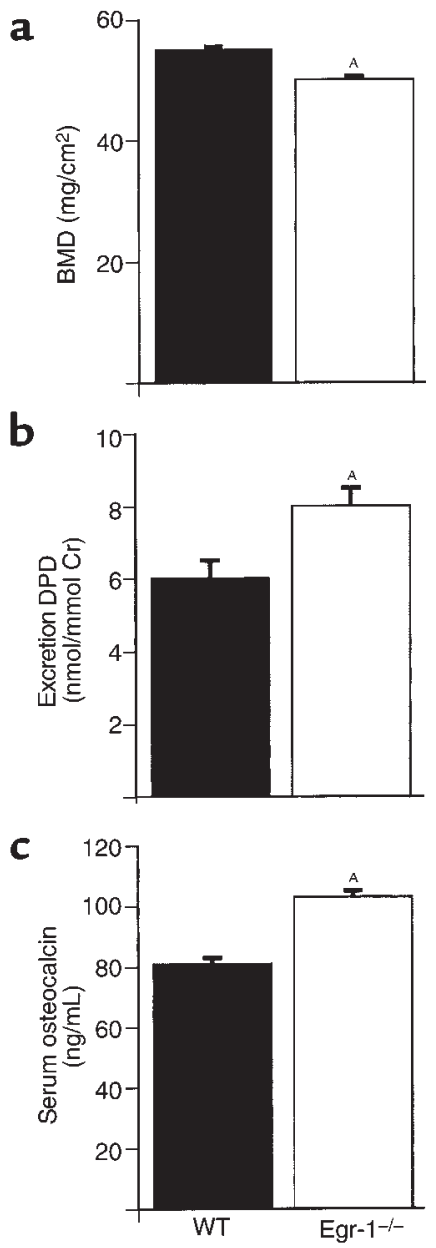

Figure 4

(a) BMD, (b) DPD excretion, and (c) osteocalcin levels in adult Egr$1^{-1-}$ mice and control WT littermates (mean \pm SEM). Measurements were carried out in samples ( $n=30$ per group) from 4-month-old intact mice. ${ }^{A} P<0.05$ compared with WT mice. 


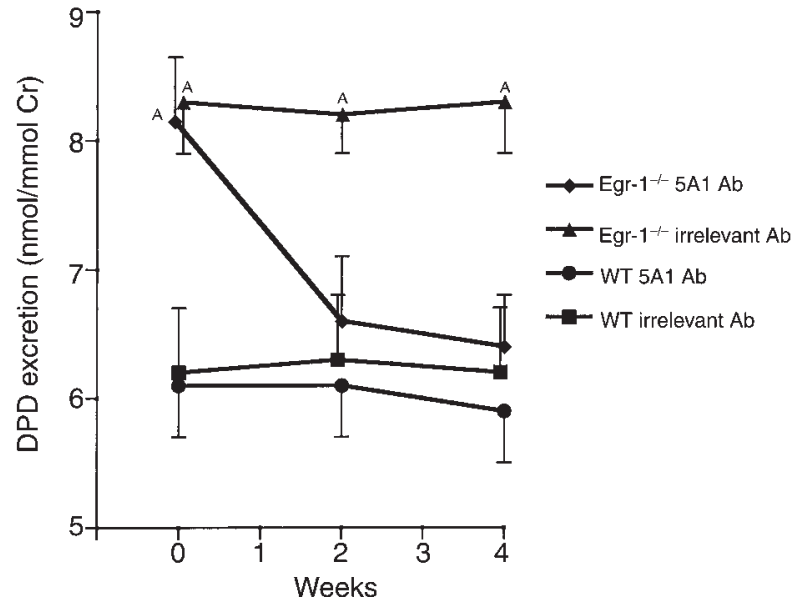

Figure 5

Treatment with $5 \mathrm{~A} 1 \mathrm{Ab}$ decreases DPD excretion in Egr-1-deficient mice. Intact adult WT and Egr-1/- mice were treated with either 5A1 $A b$ or irrelevant $A b$ for 4 weeks as described above. Results (mean + SEM) are expressed as absolute values ( $n=5$ mice per group). AP $<0.05$ compared with both groups of WT mice.

in Egr-1/- mice than in WT controls. Whereas irrelevant $\mathrm{Ab}$ had no effect in both groups of mice, treatment with $5 \mathrm{~A} 1 \mathrm{Ab}$ (Figure 5) did not alter DPD excretion in WT mice, but resulted in a significant decrease in DPD excretion in Egr-1-deficient mice. Thus, at the end of the study, Egr-1//- mice treated with $5 \mathrm{~A} 1 \mathrm{Ab}$ had a DPD excretion similar to that of WT mice treated with either irrelevant $\mathrm{Ab}$ or $5 \mathrm{~A} 1 \mathrm{Ab}$. These findings demonstrate that increased $\mathrm{M}$-CSF production is responsible for the enhanced bone resorption observed in Egr-1-deficient mice.

Ovx fails to induce bone loss and increase bone turnover in Egr-1-deficient mice. In WT mice ovx is followed by a rapid increase in stromal cell M-CSF production (12). In contrast, in Egr-1/- mice M-CSF production is maximally stimulated in both E2 replete and ovx mice (12). Thus, to further investigate the relevance of M-CSF as a mediator of the effects of E2 deficiency in bone, Egr-1/- mice and control littermates were either ovx or sham-operated, and BMD measured 2 and 4 weeks after surgery.

Before surgery, the 3 groups of WT mice had similar $\mathrm{BMD}$ values (Table 2). The 3 groups of Egr-1/- mice were also homogeneous with respect to baseline BMD. As shown in Figure 6 and Table 3, in untreated WT mice BMD decreased rapidly after ovx. Four weeks after surgery, BMD was $10.7 \%$ lower than at baseline $(P<0.05)$. In contrast, no significant changes took place in the BMD of both E2-treated ovx ( $+2.2 \%$ compared with baseline, $P$ $=\mathrm{NS})$ and sham-operated WT mice $(-1.3 \%$ compared with baseline, $P=\mathrm{NS}$ ). As a result, at 4 weeks BMD was lower $(P<0.01)$ in untreated ovx WT mice as opposed to E2-replete WT animals. Conversely, in Egr-1-/- mice no significant changes in BMD occurred after surgery in either the untreated ovx $(-1.5 \%$ compared with baseline, $P=\mathrm{NS})$, the E2-treated ovx ( $-0.1 \%$ compared with baseline, $P=\mathrm{NS})$, or the sham-operated animals $(0.6 \% \mathrm{com}$ pared with baseline, $P=\mathrm{NS}$ ). Thus, 4 weeks after ovx, all groups of Egr- $1^{-/-}$mice had similar $(P=\mathrm{NS})$ BMD values. These data demonstrate that Egr-1-deficient mice are protected against ovx-induced bone loss.

To determine if the lack of Egr-1 prevents the increase in bone resorption induced by ovx, DPD excretion was measured before and 2 and 4 weeks after ovx. At baseline, the 3 groups of WT mice and the 3 groups of Egr$1^{-/-}$mice were homogeneous with respect to DPD excretion (Table 2). In WT mice DPD excretion increased (Figure 7 and Table 3 ) by $40 \%$ in the untreated ovx group $(P<0.05)$, whereas it remained unchanged in both sham-operated $(6.0 \%$ compared with baseline, $P=$ NS) and E2-treated ovx mice $(-2.0 \%$ compared with baseline, $P=\mathrm{NS}$ ). In contrast, in Egr-1/- mice, DPD excretion did not increase in untreated ovx mice (1.3\% compared with baseline, $P=\mathrm{NS}$ ), E2-treated ovx mice (3.0\% compared with baseline, $P=\mathrm{NS}$ ), or in sham-operated mice ( $4.0 \%$ compared with baseline, $P=\mathrm{NS})$. As a result DPD levels were similar at baseline and at 4 weeks from surgery in all groups of Egr-1 $1^{-/}$mice.

To determine if Egr-1 deficiency also prevents the increase in bone formation induced by ovx, serum levels of osteocalcin were measured in samples collected at sacrifice. At 4 weeks after surgery serum osteocalcin levels were approximately $50 \%$ higher $(P<0.05)$ in WT untreated ovx mice than in both WT sham-operated and WT E2-treated ovx mice (Table 3). In contrast, in Egr-1 $1^{-/}$mice ovx was not followed by a significant increase in osteocalcin levels, a finding likely to reflect appropriate "coupling" between bone resorption and bone formation in Egr-1//- mice.

\section{Discussion}

It is now recognized that $\mathrm{E} 2$ prevents bone loss through effects on bone marrow and bone cells, which result in decreased OC formation, increased OC apoptosis, and decreased capacity of mature OCs to resorb bone (1). E2

Table 2

Baseline BMD and DPD excretion in WT and Egr-1/- mice $^{\mathrm{A}}$

\begin{tabular}{|c|c|c|c|c|c|c|}
\hline & WT sham & WT ovx & WT ovx-E2 & Egr-1-/- sham & Egr-1-/- ovx & Egr-1-/- ovx-E2 \\
\hline $\begin{array}{l}\mathrm{BMD} \\
\left(\mathrm{mg} / \mathrm{cm}^{2}\right) \\
\text { DPD }\end{array}$ & $55.6 \pm 1.0$ & $55.0 \pm 1.0$ & $54.4 \pm 1.5$ & $51.1 \pm 1.4$ & $49.6 \pm 4.4$ & $49.3 \pm 1.5$ \\
\hline$(\mathrm{nmol} / \mathrm{mmol} \mathrm{Cr})$ & $6.2 \pm 0.3$ & $6.1 \pm 0.3$ & $5.9 \pm 0.4$ & $7.9 \pm 0.4$ & $8.2 \pm 0.3$ & $8.0 \pm 0.3$ \\
\hline
\end{tabular}




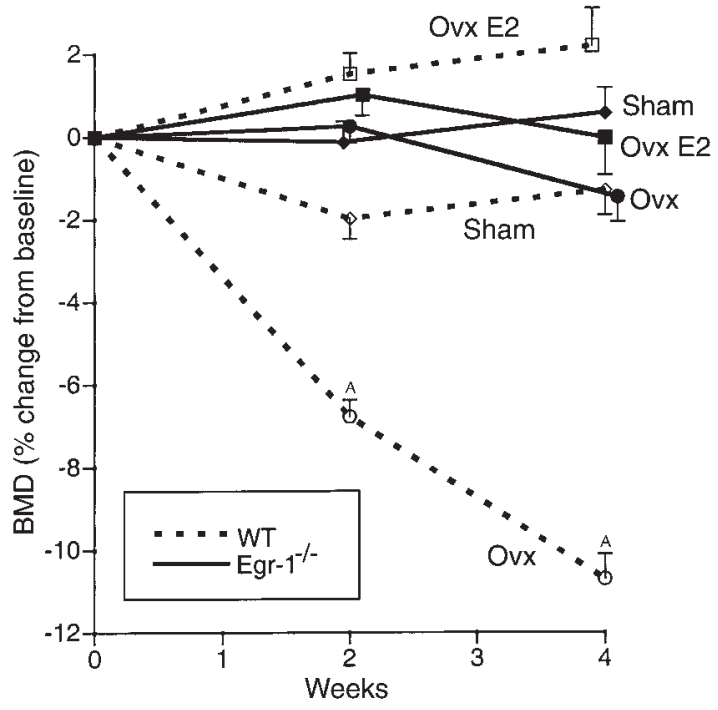

Figure 6

Egr-1-deficient mice are protected against ovx-induced bone loss. Results (mean \pm SEM) are expressed as percent of change from baseline. Thirty Egr-1//- mice and 30 control WT littermates were either ovx or sham-operated. Ovx mice were treated with either vehicle or E2 for 4 weeks. Sham-operated mice were left untreated ( $n=10$ mice per group). In vivo measurements of rear-limb BMD were carried out as described in Figure 2. ${ }^{A} P<0.05$ compared with baseline and to any other group.

modulates OC apoptosis and OC activity both directly $(34,35)$ and indirectly, through regulation of growth factors and prostaglandins $(36,37)$. Conversely, inhibition of OC formation results primarily from the ability of $\mathrm{E} 2$ to regulate the production of pro- and anti-osteoclastogenic cytokines. Among them are IL-1, IL-6, TNF, M-CSF, and osteoprotegerin $(1,11,16,17,19)$.

The current study was designed to examine the contribution of M-CSF to the bone sparing effect of E2. Several splice variants of M-CSF have been described, including a soluble and a membrane-bound form (38). Both forms are produced by bone marrow SC, a cell lineage that also produces the membrane-bound cytokine OPGL (39). By interacting with specific receptors expressed on hematopoietic precursors of the monocytic lineage, SC produced OPGL and M-CSF induced OC formation (3-5). Thus, M-CSF is one of the two currently recognized essential inducers of osteoclastogenesis.

E2 is known to downregulate both membrane-bound and soluble M-CSF, although different mechanisms are involved. The expression of membrane-bound M-CSF on bone marrow cells is directly inhibited by E2 (13, 14), although it has not yet been determined whether $\mathrm{E} 2$ regulates the SC production of membrane-bound M-CSF. In contrast, E2 does not directly downregulate the secretion of soluble M-CSF by mature SC, but rather directs the differentiation of SC precursors toward a mature phenotype characterized by a lower production of soluble M-CSF $(11,12)$. Conversely, SC that differentiate in the bone marrow of E2-deficient mice, acquire the capacity to secrete larger amounts of soluble M-CSF. These "high M-CSF-producing SC" are characterized by increased CKII activity, a phenomenon leading to increased phosphorylation of the nuclear protein Egr-1. Phosphorylated Egr-1 binds less avidly to the transcriptional activator Sp-1 and the resulting higher levels of free Sp-1 stimulate transactivation of the M-CSF gene (12). Thus, the ability of E2 to lead to the formation of SC producing low amounts of M-CSF is a key mechanism by which E2 blocks OC formation.

In this study we have examined the contribution of soluble and membrane-bound M-CSF to the pathogenesis of ovx-induced bone loss by determining whether inhibiting M-CSF function in vivo through treatment by anti-M-CSF Ab 5A1 (21) prevents the increase in bone resorption and the bone loss induced by ovx. At the dose used in this study ( $0.5 \mathrm{mg} /$ week) $5 \mathrm{~A} 1 \mathrm{Ab}$ is known to neutralize the biological functions of M-CSF in vivo (22), a finding confirmed in the current study. Osteoclastic bone resorption was assessed by bone histomorphometry and by measuring the urinary excretion of DPD, a specific and sensitive marker of bone resorption, using an assay that has been validated previously in the mouse (40). Bone density was measured in vivo by DEXA using a modification of the method described by Weinstein et al. (41). The high in vivo reproducibility of this method (2\%) makes it possible to detect significant changes in BMD as early as 1 week after ovx. This technique is therefore suitable for determining the effects of cytokine neutralization on bone density in vivo.

We found that in vivo treatment with $5 \mathrm{~A} 1 \mathrm{Ab}$ prevents the bone loss and the increase in OC number, bone resorption, and bone formation induced by ovx. These findings demonstrate the existence of a causal relationship between increased SC production of M-

Table 3

BMD, DPD excretion, and serum osteocalcin levels in WT and Egr-1/- mice at 4 weeks after surgery ${ }^{\mathrm{A}}$

\begin{tabular}{|c|c|c|c|c|c|c|}
\hline & WT sham & WT ovx & WT ovx-E2 & Egr-1 ${ }^{-/-}$sham & Egr-1-/- ovx & $\mathrm{Egr}-1^{-/-}$ovx-E2 \\
\hline $\begin{array}{l}\text { BMD } \\
\left(\mathrm{mg} / \mathrm{cm}^{2}\right) \\
\text { DPD }\end{array}$ & $54.9 \pm 1.2$ & $49.1 \pm 1.5^{\mathrm{B}}$ & $55.6 \pm 2.1$ & $51.4 \pm 1.7$ & $48.9 \pm 1.2$ & $49.2 \pm 2.6$ \\
\hline $\begin{array}{l}(\mathrm{nmol} / \mathrm{mmol} \mathrm{Cr}) \\
\text { Osteocalcin }\end{array}$ & $6.6 \pm 3.1$ & $8.5 \pm 4.1^{\mathrm{B}}$ & $5.8 \pm 1.5$ & $8.2 \pm 2.0$ & $8.3 \pm 2.6$ & $8.2 \pm 2.2$ \\
\hline$(\mathrm{ng} / \mathrm{mL})$ & $81.9 \pm 3$ & $121.8 \pm 8^{B}$ & $88.0 \pm 5$ & $102.6 \pm 5$ & $117.7 \pm 5$ & $105.3 \pm 6$ \\
\hline
\end{tabular}

${ }^{A}$ Mean \pm SEM. ${ }^{B} P<0.05$ compared with sham WT. 


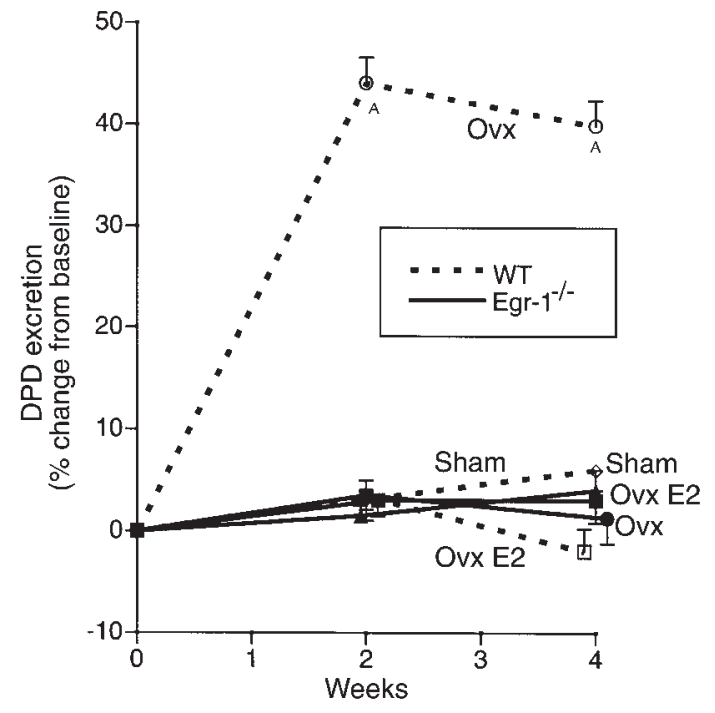

Figure 7

Ovx fails to increase DPD excretion in Egr-1/- mice. Results (mean + SEM) are expressed as percent of change from baseline. Mice were treated as described in Figure 6 ( $n=10$ mice per group). ${ }^{A} P<0.05$ compared with baseline and to any other group.

CSF and ovx-induced bone loss. Since ovx increases and $5 \mathrm{~A} 1 \mathrm{Ab}$ neutralizes both soluble and membrane-bound M-CSF, it is likely that both forms of M-CSF contribute to the pathogenesis of ovx-induced bone loss.

It is now recognized that in the mouse $\mathrm{E} 2$ replacement causes a net increase in bone density by stimulating formation on endosteal bone surfaces independently of resorption $(32,42,43)$. E2 replacement is also known to reverse the increase in osteocalcin levels induced by ovx $(18,44-46)$. The latter phenomenon reflects suppression of turnover at sites of active remodeling on cancellous bone surfaces (45). Confirming these earlier reports, in this study we found that E2 replacement led to a net increase in bone density whereas it lowered cancellous bone formation and osteocalcin levels, as measured using an assay previously validated in the mouse $(33,40)$.

Similar to E2, treatment with $5 \mathrm{~A} 1 \mathrm{Ab}$ prevented the increase in both bone resorption and cancellous bone formation. Thus, the ability of $5 \mathrm{~A} 1 \mathrm{Ab}$ to prevent bone loss is likely to be entirely related to suppression of bone resorption. However, M-CSF neutralization neither resulted in a net increase in bone density nor prevented the increase in osteocalcin induced by ovx. The inability of $5 \mathrm{~A} 1 \mathrm{Ab}$ to cause a net increase in bone density indicates the lack of a cause-effect relationship between M-CSF and the stimulatory effects of E2 on endosteal bone formation. Moreover, since the increase in osteocalcin induced by ovx is due to unregulated osteoblastogenesis (33), the failure of M-CSF neutralization to normalize osteocalcin levels suggests that ovx stimulates osteoblast proliferation through a MCSF-independent mechanism.

The relevance of M-CSF as inducer of bone loss in E2deficient mice was confirmed using Egr-1 knock-out mice. We have previously reported that M-CSF levels and OC formation are maximally stimulated in E2replete Egr-1-deficient mice (12). In the current study we have found that Egr-1-deficient mice have increased bone turnover and that M-CSF neutralization restores a normal rate of bone resorption. Importantly, $5 \mathrm{~A} 1 \mathrm{Ab}$ did not decrease DPD excretion in WT mice, a finding that confirms previous reports demonstrating that in adult mice lacking M-CSF there is a compensatory increase in the levels of cytokines that substitute for MCSF, such as IL-3, GM-CSF, and VEGF, thus assuring the renewal of the osteoclastic population $(47,48)$. We recognize that we have not excluded the possibility that Egr-1 may regulate additional factors relevant for osteoclastogenesis and bone resorption; however, since $\mathrm{M}-\mathrm{CSF}$ neutralization normalizes bone resorption in Egr-1 $1^{-/-}$mice, the data demonstrate that M-CSF plays an essential role in stimulating osteoclastogenesis above baseline levels.

We also found that in adult Egr-1/- mice BMD is approximately $10 \%$ lower than age-matched WT littermates of identical genetic background. It could be argued that the chronic overproduction of M-CSF characteristic of Egr-1-/- mice should have resulted in the finding of even lower BMD values in these mice, compared with control littermates. However, it should be noted that in ovx rats and mice long-term stimulation of osteoclastogenesis leads to a compensatory increase in bone formation that limits the net loss of bone. This hypothesis is supported by the finding of higher osteocalcin levels in Egr-1/- than in WT mice. In addition, chronic M-CSF stimulation of mature OCs markedly downregulates the expression of M-CSF receptors on these cells (49). Thus, it is conceivable that decreased responsiveness to M-CSF may contribute to explain the lack of a lower BMD in mature Egr-1-deficient mice.

In Egr-1-deficient mice ovx is unable to further increase both the SC production of M-CSF and the formation of OCs in cultures of bone marrow cells (12). Whereas ovx was followed by rapid bone loss in WT littermates, no significant decrease in BMD was detected in Egr-1/- mice. These findings are not unexpected, because the production of M-CSF and osteoclastogenesis are maximally stimulated in both E2-replete and E2-deficient Egr-1/- mice (12). As a result, ovx does not induce increases over baseline in M-CSF and OC production, a phenomenon that explains why ovx does not cause bone loss in $\mathrm{Egr}-1^{-/-}$ mice. Likewise, the lack of change in BMD in response to E2 replacement reflects the inability of E2 to repress M-CSF production and osteoclastogenesis in an Egr-1/-- background.

In summary, since $\mathrm{E} 2$ downregulates the SC production of M-CSF and ovx-induced bone loss is prevented by both M-CSF neutralization and Egr-1 deletion, Egr1-regulated M-CSF production plays a key role in the mechanism by which E2 deficiency increases bone resorption and causes bone loss. 


\section{Acknowledgments}

This study was supported, in part, by grants from the National Institutes of Health (AR-41412 and AG-13534).

1. Manolagas, S.C., and Jilka, R.L. 1995. Bone marrow, cytokines, and bone remodeling. N. Engl. J. Med. 332:305-311.

2. Suda, T., et al. 1999. Modulation of osteoclast differentiation and function by the new members of the tumor necrosis factor receptor and ligand families. Endocr. Rev. 20:345-357.

3. Lacey, D.L., et al. 1998. Osteoprotegerin ligand is a cytokine that regulates osteoclast differentiation and activation. Cell. 93:165-176.

4. Kong, Y.Y., et al. 1999. OPGL is a key regulator of osteoclastogenesis, lymphocyte development and lymph-node organogenesis. Nature. 397:315-323.

5. Yasuda, H., et al. 1998. Osteoclast differentiation factor is a ligand for osteoprotegerin/osteoclastogenesis-inhibitory factor and is identical to TRANCE/RANKL. Proc. Natl. Acad. Sci. USA. 95:3597-3602.

6. Macdonald, B.R., et al. 1986. Effects of human recombinant CSF-GM and highly purified CSF-1 on the formation of multi-nucleated cells with osteoclast characteristics in long-term bone marrow cultures. $J$. Bone Miner. Res. 1:227-232.

7. Suda, T., Takahashi, N., and Martin, T.J. 1992. Modulation of osteoclast differentiation. Endocr. Rev. 13:66-80.

8. Tanaka, S., et al. 1993. Macrophage colony-stimulating factor is indispensable for both proliferation and differentiation of osteoclast progenitors. J. Clin. Invest. 91:257-263.

9. Yoshida, H.S., et al. 1990. The murine mutation osteopetrosis is in the coding region of macrophage colony stimulating factor gene. Nature. 345:442-444.

10. Felix, R., Cecchini, M.G., and Fleish, H. 1990. Macrophage colony-stimulating factor restores in vivo bone resorption in the op/op osteopetrotic mouse. Endocrinology. 127:2592-2597.

11. Kimble, R.B., Srivastava, S., Ross, F.P., Matayoshi, A., and Pacifici, R 1996. Estrogen deficiency increases the ability of stromal cells to support osteoclastogenesis via an IL-1 and TNF mediated stimulation of MCSF production. J. Biol. Chem. 271:28890-28897.

12. Srivastava, S., et al. 1998. Estrogen blocks M-CSF gene expression and osteoclast formation by regulating phosphorylation of Egr-1 and iots interaction with $\mathrm{Sp}-1$. J. Clin. Invest. 102:1850-1859.

13. Sarma, U., Edwards, M., Motoyoshi, K., and Flanagan, A.M. 1998. Inhibition of bone resorption by 17 beta-estradiol in human bone marrow cultures. J. Cell. Physiol. 175:99-108.

14. Lea, C.K., Sarma, U., and Flanagan, A.M. 1999. Macrophage colony stimulating-factor transcripts are differentially regulated in rat bone-marrow by gender hormones. Endocrinology. 140:273-279.

15. Fuller, K., et al. 1993. Macrophage colony-stimulating factor stimulates survival and chemotactic behavior in isolated osteoclasts. J. Exp. Med. 178:1733-1744

16. Pacifici, R. 1996. Estrogen, cytokines and pathogenesis of postmenopausal osteoporosis. J. Bone Miner. Res. 11:1043-1051.

17. Lorenzo, J.A., et al. 1998. Mice lacking the type I interleukin-1 receptor do not lose bone mass after ovariectomy. Endocrinology. 139:3022-3025.

18. Ammann, P., et al. 1997. Transgenic mice expressing soluble tumor necrosis factor-receptor are protected against bone loss caused by estrogen deficiency. J. Clin. Invest. 99:1699-1703.

19. Hofbauer, L.C., et al. 1999. Estrogen stimulates gene expression and protein production of osteoprotegerin in human osteoblastic cells. Endocrinology. 140:4367-4370.

20. Kitazawa, R., Kimble, R.B., Vannice, J.L., Kung, V.T., and Pacifici, R. 1994. Interleukin-1 receptor antagonist and tumor necrosis factor binding protein decrease osteoclast formation and bone resorption in ovariectomized mice. J. Clin. Invest. 94:2397-2406.

21. Lokeshwar, B.L., and Lin, H.S. 1988. Development and characterization of monoclonal antibodies to murine macrophage colony-stimulating factor. J. Immunol. 141:483-488.

22. Gregory, S.H., Wing, E.J., Tweardy, D.J., Shadduck, R.K., and Lin, H.S. 1992. Primary listerial infections are exacerbated in mice administered neutralizing antibody to macrophage colony-stimulating factor. $J$. Immunol. 149:188-193.

23. Lee, S.L., Tourtellotte, L.C., Wesselschmidt, R.L., and Milbrandt, J. 1995. Growth and differentiation proceeds normally in cells deficient in the immediate early gene NGFI-A. J. Biol. Chem. 270:9971-9977.

24. Lee, S.L., et al. 1996. Luteinizing hormone deficiency and female infertility in mice lacking the transcription factor NGFI-A (Egr-1). Science.
273:1219-1221.

25. Poli, V., et al. 1994. Interleukin- 6 deficient mice are protected from bone loss caused by estrogen depletion. EMBOJ. 13:1189-1196.

26. Yamamoto, M., et al. 1998. The integrin ligand echistatin prevents bone loss in ovariectomized mice and rats. Endocrinology. 139:1411-1419.

27. Parfitt, A.M., et al. 1987. Bone histomorphometry: standardization of nomenclature, symbols, and units. Report of the ASBMR Histomorphometry Nomenclature Committee. J. Bone Miner. Res. 2:595-610.

28. Uebelhart, D., Gineyts, E., Chapuy, M.C., and Delmas, P.D. 1990. Urinary excretion of pyridinium crosslinks: a new marker of bone resorption in metabolic bone disease. Bone Miner. 8:87-96.

29. Seyedin, S.M., et al. 1993. Immunoassay of urinary pyridinoline: the new marker of bone resorption. J. Bone Miner. Res. 8:635-641.

30. Delmas, P.D., Gineyts, E., Bertholin, A., Garnero, P., and Marchand, F. 1993. Immunoassay of pyridoline crosslink excretion in normal adults and in Paget's disease. J. Bone Miner. Res. 8:643-648.

31. Mosmann, T. 1983. Rapid colorimetric assay for cellular growth and survival: application to proliferation and cytotoxicity assays. J. Immunol. Methods. 65:55-63.

32. Edwards, M.W., Bain, S.D., Bailey, M.C., Lantry, M.M., and Howard, G.A. 1992. 17 beta estradiol stimulation of endosteal bone formation in the ovariectomized mouse: an animal model for the evaluation of bone-targeted estrogens. Bone. 13:29-34.

33. Jilka, R.L., et al. 1998. Loss of estrogen upregulates osteoblastogenesis in the murine bone marrow. Evidence for autonomy from factors released during bone resorption. J. Clin. Invest. 101:1942-1950.

34. Kameda, T., et al. 1997. Estrogen inhibits bone resorption by directly inducing apoptosis of the bone-resorbing osteoclasts. J. Exp. Med. 186:489-495

35. Oursler, M.J., Osdoby, P., Pyfferoen, J., Riggs, B.L., and Spelsberg, T.C. 1991. Avian osteoclasts as estrogen target cells. Proc. Natl. Acad. Sci. USA. 88:6613-6617.

36. Hughes, D.E., et al. 1996. Estrogen promotes apoptosis of murine osteoclasts mediated by TGF-beta. Nat. Med. 2:1132-1136.

37. Kawaguchi, H., et al. 1995. Ovariectomy enhances and estrogen replacement inhibits the activity of bone marrow factors that stimulate prostaglandin production in cultured mouse calvariae. J. Clin. Invest. 96:539-548

38. Rajavashisth, T.B., et al. 1987. Cloning and tissue-specific expression of mouse macrophage colony-stimulating factor mRNA. Proc. Natl. Acad. Sci. USA. 84:1157-1161.

39. Suda, T., Nakamura, I., Jimi, E., and Takahashi, N. 1997. Regulation of osteoclast function. J. Bone Miner. Res. 12:869-879.

40. Blanque, R., Cottereaux, C., and Gardner, C.R. 1998. Increases in osteocalcin after ovariectomy are amplified by LPS injection: strain differences in bone remodelling. Gen. Pharmacol. 30:51-56.

41. Weinstein, R.S., Jilka, R.L., Parfitt, A.M., and Manolagas, S.C. 1997. The effects of androgen deficiency on murine bone remodeling and bone mineral density are mediated via cells of the osteoblastic lineage. Endocrinology. 138:4013-4021.

42. Liu, C.C., and Howard, G.A. 1991. Bone-cell changes in estrogen-induced bone-mass increase in mice: dissociation of osteoclasts from bone surfaces. Anat. Rec. 229:240-250.

43. Bain, S.D., Bailey, S.C., Celino, D.L., Lantry, M.M., and Edwards, M.W. 1993. High-dose estrogen inhibits bone resorption and stimulates bone formation in the ovariectomized mouse. J. Bone Miner. Res. 8:435-442.

44. Pacifici, R., et al. 1991. Effect of surgical menopause and estrogen replacement on cytokine release from human blood mononuclear cells. Proc. Natl. Acad. Sci. USA. 88:5134-5138.

45. Kimble, R.B., et al. 1994. Interleukin-1 receptor antagonist decreases bone loss and bone resorption in ovariectomized rats. J. Clin. Invest. 93:1959-1967.

46. Williams, D.C., Paul, D.C., and Black, L.J. 1991. Effects of estrogen and tamoxifen on serum osteocalcin levels in ovariectomized rats. Bone Miner. 14:205-220

47. Myint, Y.Y., et al. 1999. Granulocyte/macrophage colony-stimulating factor and interleukin-3 correct osteopetrosis in mice with osteopetrois mutation. Am. J. Pathol. 154:553-566.

48. Niida, S., et al. 1999. Vascular endothelial growth factor can substitute for macrophage colony-stimulating factor in the support of osteoclastic bone resorption. J. Exp. Med. 190:293-298.

49. Amano, H., Hofstetter, W., Cecchini, M.G., Fleisch, H., and Felix, R. 1995. Downregulation of colony-stimulating factor-1 (CSF-1) binding by CSF1 in isolated osteoclasts. Calcif. Tissue Int. 57:367-370. 